\title{
Effect of Packaging Material and Storage Period on Physico-Chemical Attributes of Osmotic Dehydrated Ripe Sapota Slices
}

\author{
Tejib Tripura ${ }^{1}$, Tapas Sarkar ${ }^{2 *}$, Madhavi Meduri $^{4}$ and Nilanjana Datta ${ }^{3}$ \\ ${ }^{1}$ Department of Fruit Science, Dr. Y. S. R. Horticulture University, Rajendranagar, \\ Hyderabad, Telangana, India \\ ${ }^{2}$ Department of Fruits Science, ${ }^{3}$ Department of Spices and Plantation Crops, \\ Bidhan Chandra Krishi Viswavidyalaya, W.B., India \\ ${ }^{4}$ Department of Fruit Science, College of Horticulture, Mojerla, \\ Mahaboobnagar, Telangana, India \\ *Corresponding author
}

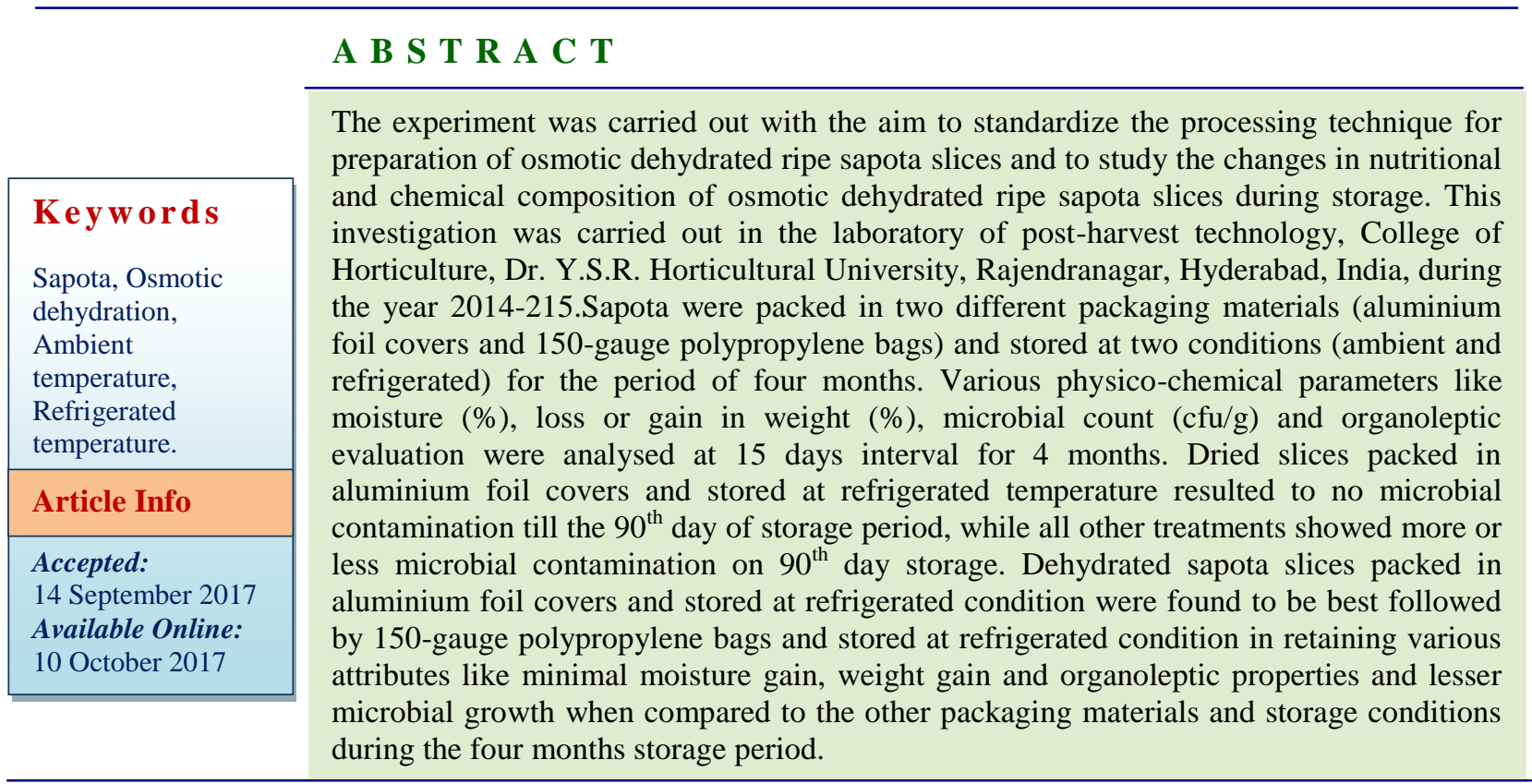

\section{Introduction}

Sapota (Achraszapota L.) is one of the most important tropical fruit. Sapota fruit is a good source of digestible sugar, virtually a treasure of minerals such as iron and calcium. The fruits have an appreciable amount of protein, fat, fibre, calcium, phosphorus, iron, carotene and vitamin $\mathrm{C}$ and also rich in bio-iron. It is growing in an area of 177.0 thousand hectares with a production of 1744.3 million tonnes (Indian Horticulture Database- 2014, NHB). It has been observed that when there is a bumper production of sapota, the fruit goes as waste for want of suitable preservation facilities. In sapota, post-harvest losses ranged from 25-30 per cent. Sapota is generally consumed as a fresh fruit. Ripened sapota 
cannot be stored for more than a day or two as it is highly perishable in nature, ripens faster and becomes unfit for consumption very soon. Among the various preservation methods, drying is the most convenient and simplest method throughout the world. Besides preserving seasonal commodities, dehydrated fruit products have inherent advantages, such as prolonged shelf life, higher degree of resistance to bacterial attack and lower transportation, handling and storage costs. The methods and the variables of drying, influence both the quality and physicochemical characteristics of the dried products (Krokida and Maroulis, 1997).

Osmotic dehydration has received greater attention in recent years as an effective method with retention of their initial fruit characteristics viz. colour, aroma, and nutritional compounds. Osmotic parameters like sugar gain and water loss are correlated with osmosis time. It involves the dehydration of fruit slices in two stages, removal of water using sugar syrup as osmotic agent and subsequent dehydration in the drier where osmotic content is further reduced to about 15 $\%$ to make the product shelf stable.

The shelf life of dehydrated ripe sapota slices depends on different factors like packaging material and storage temperature. The extension in storage life is possible by checking increase in moisture, retention of physico chemical attributes and preventing microbial activity etc. Hence, there is a need to develop a low cost technology for processing sapota fruits into value added products. Therefore, the present investigation was carried out to study the changes in physico-chemical composition of osmotic dehydrated ripe sapota slices during storage.

\section{Materials and Methods}

The investigation was carried out in the laboratory of post-harvest technology,
College of Horticulture, Dr. Y.S.R. Horticultural University, Rajendranagar, Hyderabad, India, during the year 2014-2015. Three Packaging materials Aluminum foil covers $\left(\mathrm{T}_{1}\right), 150$ gauge Polypropylene bags $\left(\mathrm{T}_{2}\right)$, Control [without package] $\left(\mathrm{C}_{1}\right)$, and two Storage conditions Ambient temperature $\left[25 \pm 2^{\circ} \mathrm{C}\right]\left(\mathrm{S}_{1}\right)$ and Refrigerated temperature [8 - $\left.10^{0} \mathrm{C}\right]\left(\mathrm{S}_{2}\right)$ were included in this investigation. There were altogether six treatments. The experiment was laid out in CRD with factorial concept with four replications.

The observation on Moisture content (\%), Loss or gain in weight (\%), Microbial load $(\mathrm{cfu} / \mathrm{ml})$ and Oganoleptic evaluation were taken at 15 days interval for 4 months. Different parameters taken are as follows

\section{Moisture (\%)}

Moisture content of fresh fruit as well as dehydrated slices was determined on percentage basis.

Dehydrated sapota slices weighing 10 grams were taken in a pre-weighed china dish and kept in a hot air oven at $70^{\circ} \mathrm{C}$ for 48 hours and then the weight was recorded using electronic balance. The dry weight was recorded until a constant weight was attained.

Moisture content was determined on fresh weight basis (Ranganna, 1986).

Loss in weight of sample on drying $(\mathrm{g})$ Moisture $(\%)=\longrightarrow$ X 100

Weight of sample taken $(\mathrm{g})$

\section{Gain in weight $(\%)$}

Loss or gain in weight of

Dehydrated slices (g)

Loss or gain

In weight $(\%)=\frac{}{\text { Weight of fresh slices }(\mathrm{g})}$ X 100 


\section{Microbial load (cfu/ml)}

\section{Total bacterial count}

For estimation of microbial count in different sample products, dilution plate method was followed (Cruik Shank et al., 1975). 1gram of sample was thoroughly mixed in $9 \mathrm{ml}$ of sterile water. $1 \mathrm{ml}$ of sample was transferred through a sterile pipette to a screw cap tube containing $9 \mathrm{ml}$ of sterile water. This gave dilution of $10^{-2}$. Similarly serial dilutions were made, $1 \mathrm{ml}$ of the serially diluted sample was placed in the sterile petridish to which cooled plate count agar medium was added and mixed thoroughly with the suspension and then allowed to set and then incubated at $28 \pm$ $2^{0} \mathrm{C}$ for 48 hours. Individual colonies were counted by using digital colony counter (light box and pen method) and multiplied with the dilution factor to get the microbial population in $1 \mathrm{ml}$ of sample.

$\mathrm{CFU} / \mathrm{ml}=\frac{\text { Number of colonies per } \mathrm{ml} \text { plate }}{\text { Total dilution factor }}$

\section{Total mould count}

Dilution plate method was followed for estimation of mould population as mentioned above in the total bacterial count (Cruik Shank et al., 1975). Potato dextrose agar medium was used for estimating the yeast and mould population. Samples were diluted in the sterile water before plating.

\section{Organoleptic evaluation}

Dried slices prepared under varied pretreatments was evaluated for sensory characteristics viz.,colour, appearance, texture, flavour, taste and overall acceptability at Hedonic 9- point rating scale (Amerine et al., 1965). Each attribute was given a separate score of 9 points. From the quality point of view, higher product scoring was treated as more acceptable. The sensory attributes were evaluated as per the format of hedonic 9rating scale given below.

\section{Packaging and storage}

The dehydrated sapota slices were packed in aluminium foil covers and 150-gauge polypropylene bags respectively and were sealed using heat impulse sealer. The packages were stored under ambient temperature $\left(25 \pm 2^{0} \mathrm{C}\right)$ and refrigerated temperature $\left(8-10^{\circ} \mathrm{C}\right)$ respectively for 4 months.

\section{Statistical analysis}

The data were subjected to statistical analysis as per the procedure outlined by Panse and Sukhatme (1985). The experiment design was Complete randomized design with factorial concept. Three replicates were maintained for each analysis, and the data from the four replicates were processed by one-way analysis of variance using the least significant test to determine the level of significance at $\mathrm{P}>0.05$.

\section{Results and Discussion}

Slices were soaking in invert sugar $60^{\circ}$ Brix for 8 hours of osmotic dehydrated ripe sapota slices and stored in different packaging materials. Slices were packed in two different packaging materials (aluminium foil covers and 150-gauge polypropylene bags) storage at ambient as well as refrigerated temperature $\left(8-10^{\circ} \mathrm{C}\right)$.

\section{Moisture (\%)}

The data pertaining to the influence of packaging materials and storage period on moisture (\%) of osmotic dehydrated ripe sapota slices are presented in table 1 . 
As it was shown in the Table 1 the moisture in the dehydrated slices were significantly affected by the treatments, storage periods and their interactions.

During the 4 months of storage period, with the increase in duration of storage period, the mean moisture content slightly increased significantly from the initial (8.00) to $120^{\text {th }}$ day (10.17). The highest mean moisture content (10.17) was observed in dehydrated slices on 120 days of storage period closely followed by 105 days storage period (10.07) and was on par with 90 days storage period (10.03), whereas, lowest mean moisture (8.37) pickup was observed on $15^{\text {th }}$ day of storage period followed by $30^{\text {th }}$ day storage period (8.94).

Results revealed that dehydrated slices packed in aluminium foil covers absorbed minimum moisture during storage as compared to 150gauge polypropylene bags. The changes in moisture content may be due to differences in water vapour permeability of the packaging film and aluminium foil having very low water vapour transmission rate. Similar findings were reported in dehydrated apple rings by Sharma and Kausal (2006) and banana chips and jackfruit chips by Molla et al., (2009).

Increase in moisture during storage period was attributed to slight pickup of moisture by osmo-dehydrated sapota slices. Analogous observation was reported by Mehta and Tomar (1980) in guava and papaya, Heikel et al., (1972) in dried mango sheets.

There was a progressive increase in moisture content of the packed upon storage. This might be due to absorption of moisture from the atmosphere by the packaging materials. Similar observation were made by Sagar and Khurdiya (1998) in dehydrated ripe mango slice, Sharma and Kausal (2006) in apple rings and banana chips and jackfruit chips by Molla et al., (2009).

\section{Gain in weight (\%)}

It is evident from the Table 2 that there were significant differences in gain in weight due to different treatments, storage period and their interactions.

Among the treatments, it was observed that the lowest mean gain in weight (1.92) was recorded in the dehydrated slices packed in aluminium foil covers stored at ambient temperature $\left(\mathrm{T}_{1} \mathrm{~S}_{1}\right)$ followed by 150 -gauge polypropylene bags stored at ambient temperature (2.02). Whereas, the highest mean gain in weight (2.75) was observed in both controls i.e. dehydrated slices stored at refrigerated temperature $\left(8-10^{\circ} \mathrm{C}\right)$ without package $\left(\mathrm{C}_{1} \mathrm{~S}_{2}\right)$ followed by $\mathrm{C}_{1} \mathrm{~S}_{1}$ - dehydrated slices stored at ambient temperature without package (2.51) during the 4 months of storage period.

\section{Bacterial count (cfu/g)}

The data in the table 3 indicates that there were significant changes in the bacterial contamination of dehydrated slices due to treatments, storage period and the interactions between them.

Significant changes in bacterial contamination were found due to the interactions between the days of storage and treatments. As the storage period increased, the bacterial contamination increased irrespective of the treatments. No bacterial contamination was noticed on the $15^{\text {th }}$ day of storage in all the treatments. On $30^{\text {th }}$ and $45^{\text {th }}$ day of storage, no contamination was observed in packed dehydrated slices except in controls- $\mathrm{C}_{1} \mathrm{~S}_{1}$ (4.37 and 9.62) and $C_{1} S_{2}$ (2.50 and 6.12) respectively. However treatments $T_{1} S_{2}$ and $\mathrm{T}_{2} \mathrm{~S}_{2}$ showed no contamination till the $90^{\text {th }}$ 
and $75^{\text {th }}$ day of storage respectively over other treatments. On $120^{\text {th }}$ day of storage, control treatments $\left(\mathrm{C}_{1} \mathrm{~S}_{1}\right.$ and $\left.\mathrm{C}_{1} \mathrm{~S}_{2}\right)$ both exhibited maximum bacterial contamination, the highest contamination was observed in control $\mathrm{C}_{1} \mathrm{~S}_{1}$ (57.75) followed by control $\mathrm{C}_{1} \mathrm{~S}_{2}$ (45.50). The minimum bacterial contamination was observed in aluminium foil covers stored at refrigerated temperature (15.37) followed by 150-gauge polypropylene bags stored at refrigerated temperature (22.50).

\section{Mould count (cfu/g)}

The data in the table 4 indicates that there were significant changes in the mould contamination of dehydrated ripe sapota slices due to treatments, days of storage and the interactions between them.

Significant changes were found due to the interactions between the days of storage and treatments. The mould count $(\mathrm{Cfu} / \mathrm{ml})$ was not found (0.00) throughout the storage period in all the treatments except in the control treatments at each successive interval of storage period. However, on the $120^{\text {th }}$ day of storage period, the mould contamination was observed in control treatments which were 2.00 and 1.25 in control $\mathrm{C}_{1} \mathrm{~S}_{1}$ and $\mathrm{C}_{1} \mathrm{~S}_{2}$ respectively. Lowest microbial load was observed in slices packed in aluminium foil covers as compared to 150-gauge polypropylene bags indicating them to be ideal material for long term storage of dehydrated sapota slices. Similar finding were recorded by Krishnaveni et al., (1999) in jackfruit bars and Evelin Mary et al., (2007) in banana powder. The storage stability may be attributed to the preservative effect of KMS and the low permeability of the packaging material to oxygen and water resulting in slower degradation of dehydrated slices hence enhancing the shelf-life.

Effect of packaging materials and storage conditions on organoleptic evaluation of osmotic dehydrated ripe sapota slices during storage

\section{Colour}

The data regarding colour of dehydrated sapota slices represented in the table 5 recorded significant variation among the treatments, duration of storage and interaction between them.

There was a significant decline in mean colour score from the initial (7.67) to $120^{\text {th }}$ day (5.84) of storage period. The colour score recorded on $15^{\text {th }}$ day was on par with $30^{\text {th }}$ day of colour score (7.47). It was observed that as the storage period increased, there was a decrease in colour score irrespective of the treatment.

\section{Hedonic 9- point rating scale}

\begin{tabular}{|c|c|c|c|c|c|c|c|c|c|c|}
\hline $\begin{array}{l}\text { Sl. } \\
\text { No. }\end{array}$ & $\begin{array}{l}\text { Sensory } \\
\text { Attributes }\end{array}$ & $\begin{array}{l}\text { Code } \\
\text { A }\end{array}$ & $\begin{array}{l}\text { Code } \\
\text { B }\end{array}$ & $\begin{array}{l}\text { Code } \\
\text { C }\end{array}$ & $\begin{array}{l}\text { Code } \\
\text { D }\end{array}$ & $\begin{array}{l}\text { Code } \\
\text { E }\end{array}$ & $\begin{array}{l}\text { Code } \\
\text { F }\end{array}$ & $\begin{array}{l}\text { Code } \\
\text { G }\end{array}$ & $\begin{array}{l}\text { Code } \\
\text { H }\end{array}$ & $\begin{array}{l}\text { Code } \\
\text { I }\end{array}$ \\
\hline 1. & Colour & & & & & & & & & \\
\hline 2. & Appearance & & & & & & & & & \\
\hline 3. & Texture & & & & & & & & & \\
\hline 4. & Taste & & & & & & & & & \\
\hline 5. & Flavor & & & & & & & & & \\
\hline 6. & $\begin{array}{l}\text { Ovarall } \\
\text { Acceptability }\end{array}$ & & & & & & & & & \\
\hline
\end{tabular}

Score: 9- Like extremely, 8- Like very much, 7- Like moderately, 6- Like slightly, 5- Neither like nor dislike, 4Dislike slightly, 3- Dislike moderately, 2- Dislike very much, 1-Dislike extremely 
Table.1 Effect of packaging materials and storage conditions on moisture (\%) content of osmotic dehydrated ripe sapota slices during storage

\begin{tabular}{|c|c|c|c|c|c|c|c|c|c|}
\hline \multirow[t]{2}{*}{ Treatments } & \multicolumn{8}{|c|}{ Days of storage } & \multirow[t]{2}{*}{ Mean } \\
\hline & 15 days & 30 days & 45 days & 60 days & 75 days & 90 days & 105 days & 120 days & \\
\hline $\mathbf{T}_{1} \mathbf{S}_{1}$ & 8.03 & 8.47 & 8.73 & 8.96 & 9.04 & 9.48 & 9.51 & 9.59 & 8.97 \\
\hline $\mathbf{T}_{1} \mathbf{S}_{2}$ & 8.03 & 8.53 & 8.72 & 9.02 & 9.28 & 9.76 & 9.96 & 10.07 & 9.17 \\
\hline $\mathbf{T}_{2} \mathbf{S}_{1}$ & 8.04 & 8.78 & 8.94 & 9.00 & 9.45 & 9.85 & 9.78 & 9.86 & 9.21 \\
\hline $\mathbf{T}_{2} \mathbf{S}_{2}$ & 8.08 & 8.85 & 9.00 & 9.02 & 9.57 & 10.15 & 10.07 & 10.18 & 9.36 \\
\hline $\mathrm{C}_{1} \mathrm{~S}_{1}$ & 9.03 & 9.46 & 9.66 & 9.77 & 9.92 & 10.37 & 10.39 & 10.50 & 9.89 \\
\hline $\mathrm{C}_{1} \mathrm{~S}_{2}$ & 9.03 & 9.58 & 9.92 & 9.72 & 10.15 & 10.57 & 10.72 & 10.80 & 10.06 \\
\hline Mean & 8.37 & 8.94 & 9.16 & 9.25 & 9.57 & 10.03 & 10.07 & 10.17 & \\
\hline \multicolumn{10}{|c|}{ F-test S.Em \pm CD at $(0.05 \%)$} \\
\hline \multicolumn{3}{|c|}{ For treatments $(\mathrm{T})$} & \multicolumn{2}{|c|}{\begin{tabular}{l|l}
$* *$ &
\end{tabular}} & \multicolumn{2}{|c|}{0.015} & \multicolumn{3}{|c|}{0.042} \\
\hline \multicolumn{2}{|c|}{ For days (D) } & & \multicolumn{2}{|l|}{$* *$} & \multicolumn{2}{|c|}{0.017} & \multicolumn{3}{|c|}{0.048} \\
\hline \multicolumn{2}{|c|}{ For D x T } & & \multicolumn{2}{|l|}{$* *$} & \multicolumn{2}{|c|}{0.042} & \multicolumn{3}{|c|}{0.119} \\
\hline
\end{tabular}

Initial value- 8.00

Table.2 Effect of packaging materials and storage conditions on gain in weight (\%) in osmotic dehydrated ripe sapota slices during storage

\begin{tabular}{|c|c|c|c|c|c|c|c|c|c|}
\hline \multirow[t]{2}{*}{ Treatments } & \multicolumn{8}{|c|}{ Days of storage } & \multirow[t]{2}{*}{ Mean } \\
\hline & 15 days & 30 days & 45 days & 60 days & 75 days & 90 days & $\begin{array}{c}105 \\
\text { days }\end{array}$ & $\begin{array}{c}120 \\
\text { days }\end{array}$ & \\
\hline $\mathbf{T}_{1} \mathbf{S}_{1}$ & 0.00 & 0.88 & 1.30 & 1.85 & 2.45 & 2.64 & 3.02 & 3.20 & 1.92 \\
\hline $\mathbf{T}_{1} \mathbf{S}_{2}$ & 0.11 & 1.15 & 1.50 & 2.07 & 2.56 & 2.94 & 3.34 & 3.56 & 2.15 \\
\hline $\mathbf{T}_{2} \mathbf{S}_{1}$ & 0.00 & 0.94 & 1.42 & 1.94 & 2.57 & 2.79 & 3.20 & 3.32 & 2.02 \\
\hline $\mathbf{T}_{2} \mathbf{S}_{2}$ & 0.23 & 1.43 & 1.71 & 2.30 & 2.72 & 3.10 & 3.45 & 3.70 & 2.33 \\
\hline $\mathrm{C}_{1} \mathrm{~S}_{1}$ & 0.21 & 1.55 & 1.91 & 2.58 & 3.03 & 3.36 & 3.57 & 3.91 & 2.51 \\
\hline $\mathrm{C}_{1} \mathrm{~S}_{2}$ & 0.27 & 1.94 & 2.50 & 2.77 & 3.17 & 3.58 & 3.76 & 4.02 & 2.75 \\
\hline Mean & 0.14 & 1.31 & 1.72 & 2.25 & 2.75 & 3.07 & 3.39 & 3.62 & \\
\hline \multicolumn{10}{|c|}{ F-test S.Em \pm CD at $(0.05 \%)$} \\
\hline \multicolumn{2}{|c|}{ For treatments $(\mathrm{T})$} & \multicolumn{3}{|c|}{\begin{tabular}{l|l}
$* *$ & \\
\end{tabular}} & \multicolumn{2}{|c|}{0.013} & \multicolumn{3}{|c|}{0.038} \\
\hline \multicolumn{2}{|c|}{ For days (D) } & \multicolumn{3}{|c|}{$* *$} & \multicolumn{2}{|c|}{0.015} & \multicolumn{3}{|c|}{0.044} \\
\hline \multicolumn{2}{|c|}{ For D x T } & \multicolumn{3}{|c|}{$* *$} & \multicolumn{2}{|c|}{0.038} & \multicolumn{3}{|c|}{0.108} \\
\hline
\end{tabular}

Initial value- 0.00

Table.3 Effect of packaging materials and storage conditions on bacterial count $(\mathrm{cfu} / \mathrm{ml})$ in osmotic dehydrated ripe sapota slices during storage

\begin{tabular}{|c|c|c|c|c|c|c|c|c|c|}
\hline \multirow[t]{2}{*}{ Treatments } & \multicolumn{8}{|c|}{ Days of storage } & \multirow[t]{2}{*}{ Mean } \\
\hline & $\begin{array}{c}15 \\
\text { days }\end{array}$ & $\begin{array}{c}\text { 30 } \\
\text { days }\end{array}$ & $\begin{array}{c}45 \\
\text { days }\end{array}$ & 60 days & 75 days & 90 days & $\begin{array}{c}105 \\
\text { days }\end{array}$ & $\begin{array}{c}120 \\
\text { days }\end{array}$ & \\
\hline $\mathbf{T}_{1} \mathbf{S}_{1}$ & 0.00 & 0.00 & 0.00 & 0.00 & 0.00 & 11.12 & 19.25 & 32.25 & 7.82 \\
\hline $\mathbf{T}_{1} \mathbf{S}_{2}$ & 0.00 & 0.00 & 0.00 & 0.00 & 0.00 & 0.00 & 10.00 & 15.37 & 3.17 \\
\hline $\mathbf{T}_{2} \mathbf{S}_{1}$ & 0.00 & 0.00 & 0.00 & 2.12 & 6.12 & 15.75 & 24.75 & 35.00 & 10.46 \\
\hline $\mathbf{T}_{2} \mathbf{S}_{2}$ & 0.00 & 0.00 & 0.00 & 0.00 & 0.00 & 10.12 & 15.50 & 22.50 & 6.01 \\
\hline $\mathrm{C}_{1} \mathrm{~S}_{1}$ & 0.00 & 4.37 & 9.62 & 16.00 & 24.75 & 35.75 & 44.25 & 57.75 & 24.06 \\
\hline $\mathrm{C}_{1} \mathrm{~S}_{2}$ & 0.00 & 2.50 & 6.12 & 12.50 & 17.00 & 24.50 & 36.25 & 45.50 & 18.04 \\
\hline Mean & 0.00 & 1.14 & 2.62 & 5.10 & 7.97 & 16.20 & 25.00 & 34.72 & \\
\hline \multicolumn{10}{|c|}{ F-test S.Em \pm CD at $(0.05 \%)$} \\
\hline \multicolumn{2}{|c|}{ For treatments $(\mathrm{T})$} & & \multicolumn{2}{|c|}{\begin{tabular}{l|l}
$* *$ &
\end{tabular}} & \multicolumn{2}{|c|}{0.224} & \multicolumn{3}{|c|}{0.627} \\
\hline \multicolumn{2}{|c|}{ For days $(\mathrm{D})$} & & \multicolumn{2}{|l|}{$* *$} & \multicolumn{2}{|c|}{0.259} & \multicolumn{3}{|c|}{0.724} \\
\hline \multicolumn{2}{|c|}{ For D x T } & & \multicolumn{2}{|l|}{$* *$} & \multicolumn{2}{|c|}{0.635} & \multicolumn{3}{|c|}{1.775} \\
\hline
\end{tabular}

Initial value- 0.00 
Table.4 Effect of packaging materials and storage conditions on mould count (cfu/ml) in osmotic dehydrated ripe sapota slices during storage

\begin{tabular}{|c|c|c|c|c|c|c|c|c|c|}
\hline \multirow[t]{2}{*}{ Treatments } & \multicolumn{8}{|c|}{ Days of storage } & \multirow[t]{2}{*}{ Mean } \\
\hline & 15 days & 30 days & 45 days & 60 days & 75 days & 90 days & $\begin{array}{c}105 \\
\text { days }\end{array}$ & $\begin{array}{c}120 \\
\text { days }\end{array}$ & \\
\hline $\mathbf{T}_{1} \mathbf{S}_{1}$ & 0.00 & 0.00 & 0.00 & 0.00 & 0.00 & 0.00 & 0.00 & 0.00 & 0.00 \\
\hline$T_{1} S_{2}$ & 0.00 & 0.00 & 0.00 & 0.00 & 0.00 & 0.00 & 0.00 & 0.00 & 0.00 \\
\hline$T_{2} S_{1}$ & 0.00 & 0.00 & 0.00 & 0.00 & 0.00 & 0.00 & 0.00 & 0.00 & 0.00 \\
\hline $\mathbf{T}_{2} \mathbf{S}_{2}$ & 0.00 & 0.00 & 0.00 & 0.00 & 0.00 & 0.00 & 0.00 & 0.00 & 0.00 \\
\hline $\mathrm{C}_{1} \mathrm{~S}_{1}$ & 0.00 & 0.00 & 0.00 & 0.00 & 0.00 & 0.00 & 0.00 & 2.00 & 0.25 \\
\hline $\mathrm{C}_{1} \mathrm{~S}_{2}$ & 0.00 & 0.00 & 0.00 & 0.00 & 0.00 & 0.00 & 0.00 & 1.25 & 0.15 \\
\hline Mean & 0.00 & 0.00 & 0.00 & 0.00 & 0.00 & 0.00 & 0.00 & 0.54 & \\
\hline \multicolumn{10}{|c|}{ F-test S.Em \pm CD at $(0.05 \%)$} \\
\hline \multicolumn{2}{|c|}{ For treatments $(\mathrm{T})$} & \multicolumn{3}{|c|}{\begin{tabular}{|l|l|}
$* *$ & \\
\end{tabular}} & \multicolumn{2}{|c|}{0.024} & \multicolumn{3}{|c|}{0.068} \\
\hline \multicolumn{2}{|c|}{ For days (D) } & \multirow{2}{*}{\multicolumn{3}{|c|}{ *** }} & \multicolumn{2}{|c|}{0.028} & \multicolumn{3}{|c|}{0.078} \\
\hline \multicolumn{2}{|c|}{ For D x T } & & & & \multicolumn{2}{|c|}{0.069} & \multicolumn{3}{|c|}{0.193} \\
\hline
\end{tabular}

Initial value- 0.00

Table.5 Effect of packaging materials and storage conditions on colour of osmotic dehydrated ripe sapota slices during storage

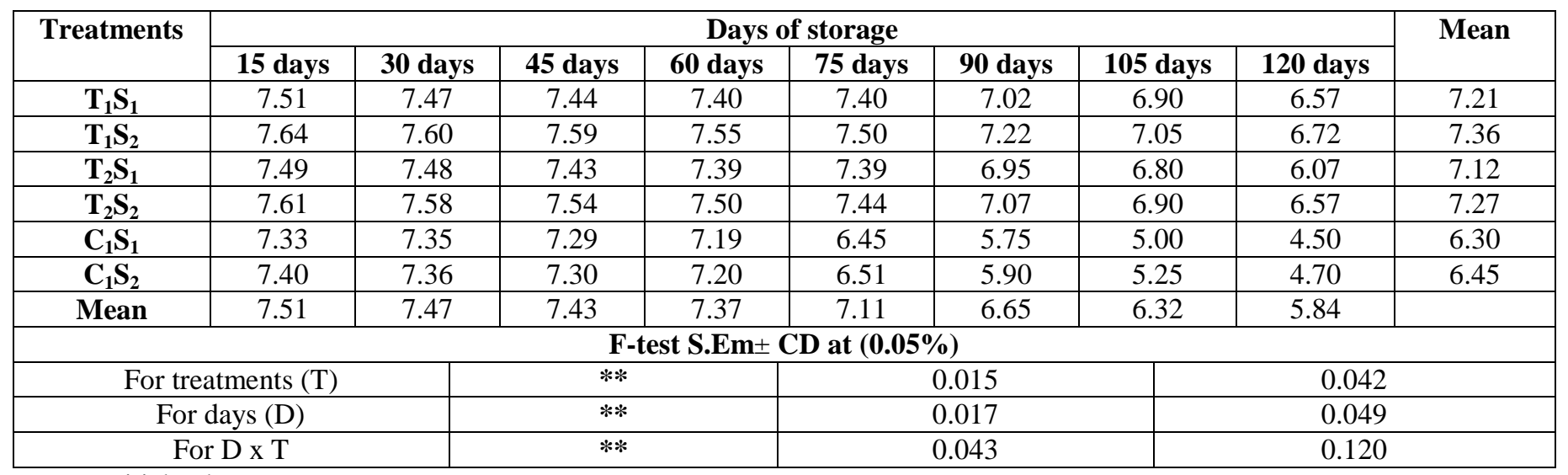

Initial value-7.67

Table.6 Effect of packaging materials and storage conditions on appearance of osmotic dehydrated ripe sapota slices during storage

\begin{tabular}{|c|c|c|c|c|c|c|c|c|c|}
\hline \multirow[t]{2}{*}{ Treatments } & \multicolumn{8}{|c|}{ Days of storage } & \multirow[t]{2}{*}{ Mean } \\
\hline & 15 days & 30 days & 45 days & 60 days & 75 days & 90 days & 105 days & 120 days & \\
\hline $\mathbf{T}_{1} \mathbf{S}_{1}$ & 7.33 & 7.00 & 6.51 & 6.07 & 5.86 & 5.54 & 5.45 & 5.35 & 5.88 \\
\hline $\mathbf{T}_{1} \mathbf{S}_{2}$ & 7.33 & 7.05 & 6.74 & 6.52 & 6.13 & 5.71 & 5.63 & 5.60 & 6.34 \\
\hline $\mathbf{T}_{2} \mathbf{S}_{1}$ & 7.33 & 6.89 & 6.51 & 6.00 & 5.68 & 5.49 & 5.41 & 5.38 & 6.08 \\
\hline $\mathbf{T}_{2} \mathbf{S}_{2}$ & 7.33 & 7.00 & 6.67 & 6.29 & 6.00 & 5.61 & 5.60 & 5.50 & 6.25 \\
\hline $\mathrm{C}_{1} \mathrm{~S}_{1}$ & 7.00 & 6.47 & 5.84 & 5.37 & 4.75 & 4.47 & 3.95 & 3.57 & 5.17 \\
\hline $\mathrm{C}_{1} \mathrm{~S}_{2}$ & 7.00 & 6.54 & 6.00 & 5.56 & 5.05 & 4.37 & 4.00 & 3.70 & 5.28 \\
\hline Mean & 7.22 & 6.82 & 6.38 & 5.97 & 5.58 & 5.20 & 5.00 & 4.85 & \\
\hline \multicolumn{10}{|c|}{ F-test S.Em \pm CD at $(0.05 \%)$} \\
\hline \multicolumn{3}{|c|}{ For treatments $(\mathrm{T})$} & \multicolumn{2}{|c|}{$* *$} & \multicolumn{2}{|c|}{0.021} & \multicolumn{3}{|c|}{0.059} \\
\hline \multicolumn{3}{|c|}{ For days (D) } & \multicolumn{2}{|c|}{$* *$} & \multicolumn{2}{|c|}{0.024} & \multicolumn{3}{|c|}{0.068} \\
\hline \multicolumn{3}{|c|}{ For D x T } & \multicolumn{2}{|c|}{$* *$} & \multicolumn{2}{|c|}{0.059} & \multicolumn{3}{|c|}{0.167} \\
\hline
\end{tabular}

Initial value-7.33 
Table.7 Effect of packaging materials and storage conditions on texture of osmotic dehydrated ripe sapota slices during storage

\begin{tabular}{|c|c|c|c|c|c|c|c|c|c|}
\hline \multirow[t]{2}{*}{ Treatments } & \multicolumn{8}{|c|}{ Days of storage } & \multirow[t]{2}{*}{ Mean } \\
\hline & 15 days & 30 days & 45 days & 60 days & 75 days & 90 days & 105 days & 120 days & \\
\hline $\mathbf{T}_{1} \mathbf{S}_{1}$ & 6.55 & 6.32 & 6.12 & 6.00 & 5.82 & 5.67 & 5.57 & 5.40 & 5.93 \\
\hline $\mathbf{T}_{1} \mathbf{S}_{2}$ & 6.66 & 6.57 & 6.50 & 6.36 & 6.15 & 6.05 & 5.92 & 5.70 & 6.24 \\
\hline $\mathbf{T}_{2} \mathbf{S}_{1}$ & 6.50 & 6.30 & 6.00 & 5.85 & 5.72 & 5.55 & 5.37 & 5.15 & 5.80 \\
\hline $\mathbf{T}_{2} \mathbf{S}_{2}$ & 6.60 & 6.41 & 6.32 & 6.15 & 6.02 & 5.85 & 5.67 & 5.52 & 6.07 \\
\hline $\mathrm{C}_{1} \mathrm{~S}_{1}$ & 6.10 & 5.65 & 5.37 & 5.00 & 4.55 & 4.30 & 3.87 & 2.62 & 4.68 \\
\hline $\mathbf{C}_{1} \mathbf{S}_{2}$ & 6.17 & 5.76 & 5.45 & 5.07 & 4.72 & 4.42 & 4.10 & 3.57 & 4.91 \\
\hline Mean & 6.43 & 6.17 & 5.96 & 5.74 & 5.50 & 5.30 & 5.08 & 4.66 & \\
\hline \multicolumn{10}{|c|}{ F-test S.Em \pm CD at $(0.05 \%)$} \\
\hline \multicolumn{3}{|c|}{ For treatments $(\mathrm{T})$} & \multicolumn{2}{|l|}{$* *$} & \multicolumn{2}{|c|}{0.046} & \multicolumn{3}{|c|}{0.130} \\
\hline \multicolumn{3}{|c|}{ For days (D) } & \multirow{2}{*}{\multicolumn{2}{|c|}{$\frac{* *}{* *}$}} & \multirow{2}{*}{\multicolumn{2}{|c|}{$\begin{array}{l}0.053 \\
0.131\end{array}$}} & \multicolumn{3}{|c|}{0.150} \\
\hline \multicolumn{3}{|c|}{ For D x T } & & & & & \multicolumn{3}{|c|}{0.368} \\
\hline
\end{tabular}

Initial value-6.67

Table.8 Effect of packaging materials and storage conditions on taste of osmotic dehydrated ripe sapota slices during storage

\begin{tabular}{|c|c|c|c|c|c|c|c|c|c|}
\hline \multirow[t]{2}{*}{ Treatments } & \multicolumn{8}{|c|}{ Days of storage } & \multirow[t]{2}{*}{ Mean } \\
\hline & 15 days & 30 days & 45 days & 60 days & 75 days & 90 days & 105 days & 120 days & \\
\hline$T_{1} S_{1}$ & 8.00 & 7.89 & 7.79 & 7.75 & 7.60 & 7.50 & 7.20 & 7.00 & 7.59 \\
\hline $\mathbf{T}_{1} \mathbf{S}_{2}$ & 8.00 & 7.94 & 7.90 & 7.82 & 7.70 & 7.58 & 7.51 & 7.27 & 7.71 \\
\hline $\mathbf{T}_{2} \mathbf{S}_{1}$ & 8.00 & 7.88 & 7.79 & 7.71 & 7.58 & 7.45 & 7.07 & 6.84 & 7.54 \\
\hline $\mathbf{T}_{2} \mathbf{S}_{2}$ & 8.00 & 7.90 & 7.82 & 7.80 & 7.63 & 7.56 & 7.38 & 7.12 & 7.65 \\
\hline $\mathrm{C}_{1} \mathrm{~S}_{1}$ & 7.65 & 7.50 & 7.40 & 7.00 & 6.12 & 5.50 & 5.00 & 4.25 & 6.30 \\
\hline $\mathrm{C}_{1} \mathrm{~S}_{2}$ & 7.70 & 7.53 & 7.42 & 7.15 & 6.25 & 5.57 & 5.20 & 4.52 & 6.42 \\
\hline Mean & 7.89 & 7.77 & 7.69 & 7.54 & 7.14 & 6.86 & 6.56 & 6.17 & \\
\hline \multicolumn{10}{|c|}{ F-test S.Em \pm CD at $(0.05 \%)$} \\
\hline \multicolumn{3}{|c|}{ For treatments $(\mathrm{T})$} & \multicolumn{2}{|c|}{$* *$} & \multicolumn{2}{|c|}{0.018} & \multicolumn{3}{|c|}{0.052} \\
\hline \multicolumn{3}{|c|}{ For days (D) } & \multicolumn{2}{|c|}{$* *$} & \multicolumn{2}{|r|}{0.021} & \multicolumn{3}{|c|}{0.060} \\
\hline \multicolumn{3}{|c|}{ For D x T } & \multicolumn{2}{|c|}{$* *$} & \multicolumn{2}{|r|}{0.052} & \multicolumn{3}{|c|}{0.148} \\
\hline
\end{tabular}

Initial value- 8.00

Table.9 Effect of packaging materials and storage conditions on flavour of osmotic dehydrated ripe sapota slices during storage

\begin{tabular}{|c|c|c|c|c|c|c|c|c|c|}
\hline \multirow[t]{2}{*}{ Treatments } & \multicolumn{8}{|c|}{ Days of storage } & \multirow[t]{2}{*}{ Mean } \\
\hline & 15 days & 30 days & 45 days & 60 days & 75 days & 90 days & 105 days & 120 days & \\
\hline $\mathbf{T}_{1} \mathbf{S}_{1}$ & 6.67 & 6.55 & 6.47 & 6.40 & 6.35 & 6.21 & 6.05 & 5.87 & 6.32 \\
\hline $\mathbf{T}_{1} \mathbf{S}_{2}$ & 6.67 & 6.62 & 6.55 & 6.48 & 6.43 & 6.33 & 6.21 & 5.95 & 6.40 \\
\hline$T_{2} S_{1}$ & 6.60 & 6.46 & 6.41 & 6.36 & 6.24 & 6.05 & 5.89 & 5.65 & 6.21 \\
\hline $\mathbf{T}_{2} \mathbf{S}_{2}$ & 6.66 & 6.57 & 6.50 & 6.43 & 6.41 & 6.25 & 6.00 & 5.62 & 6.30 \\
\hline $\mathrm{C}_{1} \mathrm{~S}_{1}$ & 6.42 & 6.12 & 5.47 & 5.02 & 4.87 & 4.57 & 4.41 & 4.00 & 5.11 \\
\hline $\mathrm{C}_{1} \mathbf{S}_{2}$ & 6.40 & 6.12 & 5.62 & 5.20 & 5.00 & 4.67 & 4.50 & 4.12 & 5.20 \\
\hline Mean & 6.57 & 6.41 & 6.17 & 5.98 & 5.88 & 5.68 & 5.51 & 5.20 & \\
\hline \multicolumn{10}{|c|}{ F-test S.Em \pm CD at $(0.05 \%)$} \\
\hline \multicolumn{3}{|c|}{ For treatments $(\mathrm{T})$} & \multicolumn{2}{|c|}{$* *$} & \multicolumn{3}{|c|}{0.013} & \multicolumn{2}{|l|}{0.038} \\
\hline \multicolumn{3}{|c|}{ For days (D) } & \multicolumn{2}{|c|}{$* *$} & \multicolumn{3}{|c|}{0.015} & \multicolumn{2}{|l|}{0.044} \\
\hline \multicolumn{3}{|c|}{ For $\mathrm{D} \times \mathrm{T}$} & \multicolumn{2}{|c|}{$* *$} & \multicolumn{3}{|c|}{0.038} & \multicolumn{2}{|l|}{0.108} \\
\hline
\end{tabular}

Initial value-6.67 
Table.10 Effect of packaging materials and storage conditions on overall acceptability of dehydrated ripe sapota slices during storage

\begin{tabular}{|c|c|c|c|c|c|c|c|c|c|}
\hline \multirow[t]{2}{*}{ Treatments } & \multicolumn{8}{|c|}{ Days of storage } & \multirow[t]{2}{*}{ Mean } \\
\hline & 15 days & 30 days & 45 days & 60 days & 75 days & 90 days & 105 days & 120 days & \\
\hline $\mathbf{T}_{1} \mathbf{S}_{1}$ & 7.33 & 7.07 & 6.95 & 6.78 & 6.60 & 6.41 & 6.33 & 6.00 & 6.68 \\
\hline $\mathbf{T}_{1} \mathbf{S}_{2}$ & 7.33 & 7.20 & 7.07 & 7.00 & 6.83 & 6.63 & 6.48 & 6.32 & 6.86 \\
\hline $\mathbf{T}_{2} \mathbf{S}_{1}$ & 7.33 & 7.00 & 6.91 & 6.67 & 6.52 & 6.37 & 6.30 & 5.92 & 6.63 \\
\hline $\mathbf{T}_{2} \mathbf{S}_{2}$ & 7.33 & 7.17 & 7.00 & 6.84 & 6.71 & 6.51 & 6.41 & 6.21 & 6.77 \\
\hline$C_{1} S_{1}$ & 7.00 & 6.50 & 6.35 & 6.02 & 5.42 & 5.00 & 4.72 & 4.25 & 5.65 \\
\hline $\mathbf{C}_{1} \mathbf{S}_{2}$ & 7.05 & 6.67 & 6.40 & 6.12 & 5.50 & 5.10 & 4.85 & 4.50 & 5.77 \\
\hline Mean & 7.22 & 6.93 & 6.78 & 6.57 & 6.26 & 6.00 & 5.85 & 5.53 & \\
\hline \multicolumn{10}{|c|}{ F-test S.Em \pm CD at $(0.05 \%)$} \\
\hline \multicolumn{3}{|c|}{ For treatments $(\mathrm{T})$} & \multicolumn{2}{|c|}{ ** } & \multicolumn{3}{|c|}{0.014} & \multicolumn{2}{|c|}{0.041} \\
\hline \multicolumn{3}{|c|}{ For days (D) } & \multicolumn{2}{|l|}{$* *$} & \multicolumn{3}{|c|}{0.017} & \multicolumn{2}{|l|}{0.047} \\
\hline \multicolumn{3}{|c|}{ For D x T } & \multicolumn{2}{|l|}{ ** } & \multicolumn{2}{|c|}{0.041} & & \multicolumn{2}{|l|}{0.117} \\
\hline
\end{tabular}

Initial value-7.33

$\mathrm{T}_{1} \mathrm{~S}_{1}$-Aluminium foil covers + Storage at ambient temperature.

$\mathrm{T}_{2} \mathrm{~S}_{1}-150$ gauge Polypropylene bags + Storage at ambient temperature.

$\mathrm{T}_{1} \mathrm{~S}_{2}$-Aluminium foil covers + Storage at refrigerated temperature $\left(8-10^{0} \mathrm{C}\right)$.

$\mathrm{T}_{2} \mathrm{~S}_{2}-150$ gauge Polypropylene bags + Storage at refrigerated temperature $\left(8-10^{\circ} \mathrm{C}\right)$.

$\mathrm{C}_{1} \mathrm{~S}_{1}$-Control without package + Storage at ambient temperature.

$\mathrm{C}_{1} \mathrm{~S}_{2}$-Control without package+Storage at refrigerated temperature $\left(8-10^{\circ} \mathrm{C}\right)$.

\section{Appearance}

The data regarding the appearance of dehydrated sapotaslices presented in the table 6 recorded significant variation among the treatments, duration of storage and interaction between them.

Among the treatments best mean appearance score (6.34) was observed in dehydrated slices packed in aluminium foil covers and stored at refrigerated temperature $\left(\mathrm{T}_{1} \mathrm{~S}_{2}\right)$ followed by packed in 150-gauge polypropylene bags and stored at refrigerated temperature $\left(\mathrm{T}_{2} \mathrm{~S}_{2}\right)$ (6.25) while least mean appearance score 5.17 was found in control with ambient temperature $\left(\mathrm{C}_{1} \mathrm{~S}_{1}\right)$ followed by with refrigerated temperature $5.28\left(\mathrm{C}_{1} \mathrm{~S}_{2}\right)$.

There was a significant decline in mean appearance score from the initial (7.33) to $120^{\text {th }}$ day (4.85) of storage period. It was observed that as the storage period increased, there was a decrease in appearance score irrespective of the treatment.

\section{Texture}

The data pertaining to texture score of dehydrated sapota slices presented in the table 7 recorded significant variation among the treatments, duration of storage and interaction between them.

Among the treatments best mean texture score (6.24) was observed in dehydrated slices packed in aluminium foil covers and stored at refrigerated temperature $\left(T_{1} S_{2}\right)$ followed by packed in 150-gauge polypropylene bags and stored at refrigerated temperature $\left(\mathrm{T}_{2} \mathrm{~S}_{2}\right)$ (6.07) while least mean texture score (4.68) was found in control with ambient temperature $\left(\mathrm{C}_{1} \mathrm{~S}_{1}\right)$ followed by (4.91) control with refrigerated temperature $\left(\mathrm{C}_{1} \mathrm{~S}_{2}\right)$.

There was a significant decline in mean texture score from the initial (6.67) to $120^{\text {th }}$ day (4.66) of storage period. It was observed that with increase in storage period, there was a decrease in texture score irrespective of the treatments. 


\section{Taste}

The data pertaining to taste score of dehydrated sapota slices represented in the table 8 recorded significant variation among the treatments, duration of storage and interaction between them.

Among the treatments best mean taste score (7.71) was observed in dehydrated slices packed in aluminium foil covers and stored at refrigerated temperature $\left(T_{1} S_{2}\right)$ followed by packed in 150-gauge polypropylene bags and stored at refrigerated temperature $\left(\mathrm{T}_{2} \mathrm{~S}_{2}\right)$ (7.65) while least taste score (6.30) was found in control with ambient temperature $\left(\mathrm{C}_{1} \mathrm{~S}_{1}\right)$ followed by (6.42) control with refrigerated temperature $\left(\mathrm{C}_{1} \mathrm{~S}_{2}\right)$.

\section{Flavour}

The data pertaining to flavour score of dehydrated sapota slices presented in the table 9 recorded significant variation among the treatments, duration of storage and interaction between them.

Among the treatments best mean flavour score (6.40) was observed in dehydrated slices packed in aluminium foil covers and stored at refrigerated temperature $\left(\mathrm{T}_{1} \mathrm{~S}_{2}\right)$ followed by packed in aluminium foil covers and stored at ambient temperature $\left(\mathrm{T}_{1} \mathrm{~S}_{1}\right)$ (6.32) and which was on par with $\mathrm{T}_{2} \mathrm{~S}_{2}$ (6.30) while least flavour score (5.11) was found in control with ambient temperature $\left(\mathrm{C}_{1} \mathrm{~S}_{1}\right)$ followed by (5.20) control with refrigerated temperature $\left(\mathrm{C}_{1} \mathrm{~S}_{2}\right)$.

There was a significant decline in mean flavour score from the initial (6.67) to $120^{\text {th }}$ day (5.20) of storage period.

It was observed that as the storage period increased, there was a decrease in flavor score irrespective of the treatment.

\section{Overall acceptability}

The data regarding overall acceptability score of dehydrated ripe sapota slices represented in the table 10 recorded significant variation among the treatments, duration of storage and interaction between them.

Among the treatments best mean overall acceptability score (6.86) was observed in dehydrated slices packed in aluminium foil covers and stored at refrigerated temperature $\left(\mathrm{T}_{1} \mathrm{~S}_{2}\right)$ followed by packed in 150-gauge polypropylene bags and stored at refrigerated temperature $\left(\mathrm{T}_{2} \mathrm{~S}_{2}\right)$ (6.77) while least overall acceptability score (5.65) was found in control with ambient temperature $\left(\mathrm{C}_{1} \mathrm{~S}_{1}\right)$ followed by (5.77) control with refrigerated temperature $\left(\mathrm{C}_{1} \mathrm{~S}_{2}\right)$.

There was a significant decline in mean overall acceptability score from the initial (7.33) to $120^{\text {th }}$ day (5.53) of storage period. It was observed that as the storage period increased, there was a decrease in mean overall acceptability score irrespective of the treatment.

There was a gradual decrease in score during the 4 months storage for colour, appearance, texture, taste, flavor and overall acceptability in all samples at ambient as well as at refrigerated storage temperature. Osmotic dehydrated sapota slices prepared using invert sugar and fructose sugar syrup gave first and second rank with highest taste score. Similar trend was observed after 4 months in slices stored at ambient and refrigerated temperature.

Refrigerated storage obtained higher score for sensory properties than those stored at ambient condition. Similar results were reported by Gawade and Waskar (2003), Deol and Bhullar (1972), Gouda et al., (1975) and Thonta and Patil (1988) for dried figs. 
The organoleptic score is varied with the treatment and packaging during storage. Osmotic followed by solar dehydrated slices packed in aluminium foil covers and stored at refrigerated temperature scores highest overall acceptability than the other treatments. The lower scores for 150-gauge polypropylene bags and stored at ambient temperature may be due to higher moisture absorption and gas permeability characteristics of the polypropylene bags. Similar results were reported by Vijayanand et al., (2000) while working on guava fruit bar.

Sensory quality of dehydrated ripe sapota slices was significantly affected during four months of storage period. Overall sensory score of slices showed a decreasing trend with advance in storage period, could be due to enzymatic and non-enzymatic oxidation process, which might have caused for reduction in sensory score of the slices. Similar findings were reported in guava slices by Mehta and Tomar (1980). Gurumeenakshi et al., (2005) also reported gradual decrease in colour and appearance of osmotic followed by solar dried papaya slices during storage. Loss of flavor during storage was also reported in papaya slices (Aruna et al., 2000).

The texture and taste score also decreased and this could be also due to the same effect. The decrease in overall acceptability of osmotic followed by solar dried sapota slices during storage was significantly evident and could be because of reduction score of all sensory parameters of sapota slices. Gurumeenakshi et al., (2005) also reported similar results on osmo-dried papaya.

\section{References}

Amerine, M. A., Pangborn, R. M. and Roesslev, E. B. 1965. Principle of Sensory Evaluation of Food. Academic
Press Inc, New York.

Aruna, K., Vimala, V. and Dhanalaxmi, K. 2000.Studies on preparation and keeping quality of papaya. Bev Food World, 27: 15-20.

Cruick Shank, Durgid, J. P., Marimon, B. P. and Surian R. H. A. 1975.Medical microbiology. The practice of medical microbiology. Churchil living stone, Edinberg, London and New York 306.

Deol, J.S., and Bhullar, D.S.1972. Effect of wrappers and growth regulators on the storage life of mango fruits. Punjab Horti. Journal, 12(2-3): 114-119.

Evelinemary, A., Jecobb john, $P$ and Vijayanand, D. 2007. Packaging and storage study on spray dried ripe banana powder under ambient conditions. Journal of Food Science Technology, 44(1) 16-21.

Gawade, M.H., and Waskar, D.P. 2003. Studies on processing and storage of fig fruits. Journal of maharastra Agric Univ., 28:188-150.

Gouda, M.S., Zouil, M.B., EL-Zalaki, E.M. and Mohamed, M. 1975. Technology studies on fig varieties planted in Arab republic of Egypt. Alexandria Journal of Agric. Res., 25:459-466.

Gurumeenakshi, G., Manimegalar, G., Maragatham, S. and Jaberaj, S. 2005. Ascorbic acid and KMS as new food additives for osmo-dried foods. $\mathrm{Bev}$ Food World, 32:50-51

Heikel, H.A., Sanafiri, N.Y and Shooman, M.A. 1972. Some factor affecting quality of dried mango sheets. Agr. Res. Rev., 50:185-194.

Krishnavedi, A., Manimegalai, G., Vennila, P. and Sravanakumar, R. 1999. Storage stability of jackfruit bar in different packaging materials. Indian Food Packer, 53: 67-71.

Krokida, M.K., and Maroulis, Z.B. 1997. Effect of drying method on shrinkage and porosity. Drying Technology, 15: 
1145-1155.

Mehta, G.L., and Tomar, M.C. 1980. Studies on dehydration of tropical fruits in Uttar Pradesh Guava: Indian food packer, 34(3): 12-15.

Molla, M.M., Nasrin T.A.A., Islam M.N. and Bhuyan A.J. 2009. Preparing and packaging of jackfruit chips. International Journal of Sustainable Crop Production, 3(6) : 41-57.

Panse, V.G., and Sukhatme, P.V. 1985. Statistical methods for agricultural workers. Indian Council of Agricultural Research, New Delhi, pp. 152-155.

Pearson, D., 1990. Pearson's Composition and Analysis of food. $8^{\text {th }}$ ed. 13.Longman Publishing Ltd. Singapore.

Ranganna, S., 1986. Handbook of analysis and quality control for fruit and vegetable products, $2^{\text {nd }}$ edn, Tata McGraw-Hill, New Delhi, India, Pp. 13, 594-645.

Sagar, V.R., and Khurdiya, D.S. 1998. Improved products from ripe mango (cv. Dashehari).Indian Food Packer, 52(6): 3-7.
2006.Evaluation of apple cultivars for dehydration.Journal Food Science Technology, 43(2):177-181.

Thonta, G.T., and Patil, V.K. 1988. Studies on drying of fig fruits.Indian Food Packer, 42:94-99.

Veda, S.K., Patel and Srinivasan, K. 2008. Influence of food acidulants and antioxidant spices on the bioaccessibility of Bita- carotene from selected vegetables. Journal of Agricultural and Food Chemistry, 56(18): 8714-8719.

Vejayanand, P., Yadav, A.R., Balasubarmanyam, N. and Narasimham, P. 2000. Storage stability of guava fruit bar prepared using a new process. LebensmWiss Technology, 33: 132137.

Vejayanand, P., Yadav, A.R., Balasubarmanyam, N. and Narasimham, P. 2000. Storage stability of guava fruit bar prepared using a new process. Lebensm Wiss Technology, 33: 132137.

Sharma, K.D., and Alkeshkausal, B.B.L.

\section{How to cite this article:}

Tejib Tripura, Tapas Sarkar, Madhavi Meduri and Nilanjana Datta. 2017. Effect of Packaging Material and Storage Period on Physico-Chemical Attributes of Osmotic Dehydrated Ripe Sapota Slices. Int.J.Curr.Microbiol.App.Sci. 6(10): 1600-1611. doi: https://doi.org/10.20546/ijcmas.2017.610.192 